\title{
Advances in HgCdTe APDs and LADAR Receivers ${ }^{1}$
}

\author{
Steven Bailey, William McKeag, Jinxue Wang, and Michael Jack \\ Raytheon Vision Systems, 75 Coromar Drive; Goleta, CA 93117 \\ Farzin Amzajerdian from NASA Langley Research Center
}

\begin{abstract}
Raytheon is developing NIR sensor chip assemblies (SCAs) for scanning and staring 3D LADAR systems. High sensitivity is obtained by integrating high performance detectors with gain i.e. APDs with very low noise Readout Integrated Circuits. Unique aspects of these designs include: independent acquisition (non-gated) of pulse returns, multiple pulse returns with both time and intensity reported to enable full 3D reconstruction of the image. Recent breakthrough in device design has resulted in $\mathrm{HgCdTe}$ APDs operating at $300 \mathrm{~K}$ with essentially no excess noise to gains in excess of 100 , low NEP $<1 \mathrm{nW}$ and $\mathrm{GHz}$ bandwidths and have demonstrated linear mode photon counting. SCAs utilizing these high performance APDs have been integrated and demonstrated excellent spatial and range resolution enabling detailed 3D imagery both at short range and long ranges. In this presentation we will review progress in high resolution scanning, staring and ultra-high sensitivity photon counting LADAR sensors.
\end{abstract}

Keywords: APD, HgCdTe, LADAR

\section{INTRODUCTION AND OUTLINE}

This paper provides an overview of advanced LADAR receiver technology at Raytheon Vision Systems ranging from high sensitivity single element APDs and associated low noise amplifiers to high speed scanning arrays $256 \times 4$ and very large format $256 \times 256$ asynchronous (non-gated) flash receivers and small format linear mode photon counting modules. These have in common the use of MBE grown $\mathrm{n}$ on $\mathrm{p} \mathrm{HgCdTe}$ engineered for excess noise factors near unity, operation at ambient temperatures and bandwidth up to $1 \mathrm{GHz}$. In this paper we focus on the development of the largest $\mathrm{HgCdTe}$ APD LADAR receiver operating at ambient and utilizing a sophisticated multiple laser pulse return ROIC. This development has been supported in part by NASA under the Autonomous Landing and Hazard Avoidance Technology program

In Section 2 we overview HgCdTe APD based LADAR sensor products at Raytheon, in section 3 the ALHAT project is described with focus on safe landing for future lunar and planetary missions, In section 4 the HgCdTe APD process and performance is summarized; section 5 introduces the advanced Readout Integrated Circuit and section 5 describes recent measurements of the hybridized Sensor Chip Assemblies (SCAs) including response uniformity, gain, NEP and MDS of the integrated receiver chip. Section 6 highlights the low profile, low power package and electronics and Section 7 summarizes and looks at future directions.

\section{LADAR SENSORS AT RAYTHEON}

Figure 1 illustrates the LADAR FPA components and associated processes.

These are primarily based on MBE engineered $\mathrm{HgCdTe}$ APDs with Fex $\sim 1$, high gain and GHz bandwidths at ambient and cryogenic temperatures. Raytheon also supports high performance LADAR sensors fabricated utilizing other devices such as InGaAs, PIN and APDs. Products include:

- Large 100mciron to 300 micron Single Element APDs for Ambient or TEC Operation in the 1-2 micron band:

- Scanning LADAR sensors $4 \times 256$ configuration;

\footnotetext{
${ }^{1}$ The Authors Acknowledge The Support from NASA's ALHAT Program Contract NNL08AA46C Program Manager is Dr. Farzin Amzajerdian
} 
- Large Format Flash Sensors $(256 \times 256)$ which provide up to 3 laser pulse returns with both range and intensity. This structure is utilized for the ALHAT sensor described below

- Photon Counting Modules with Electronics and Dewar for long range LADAR and laser communications

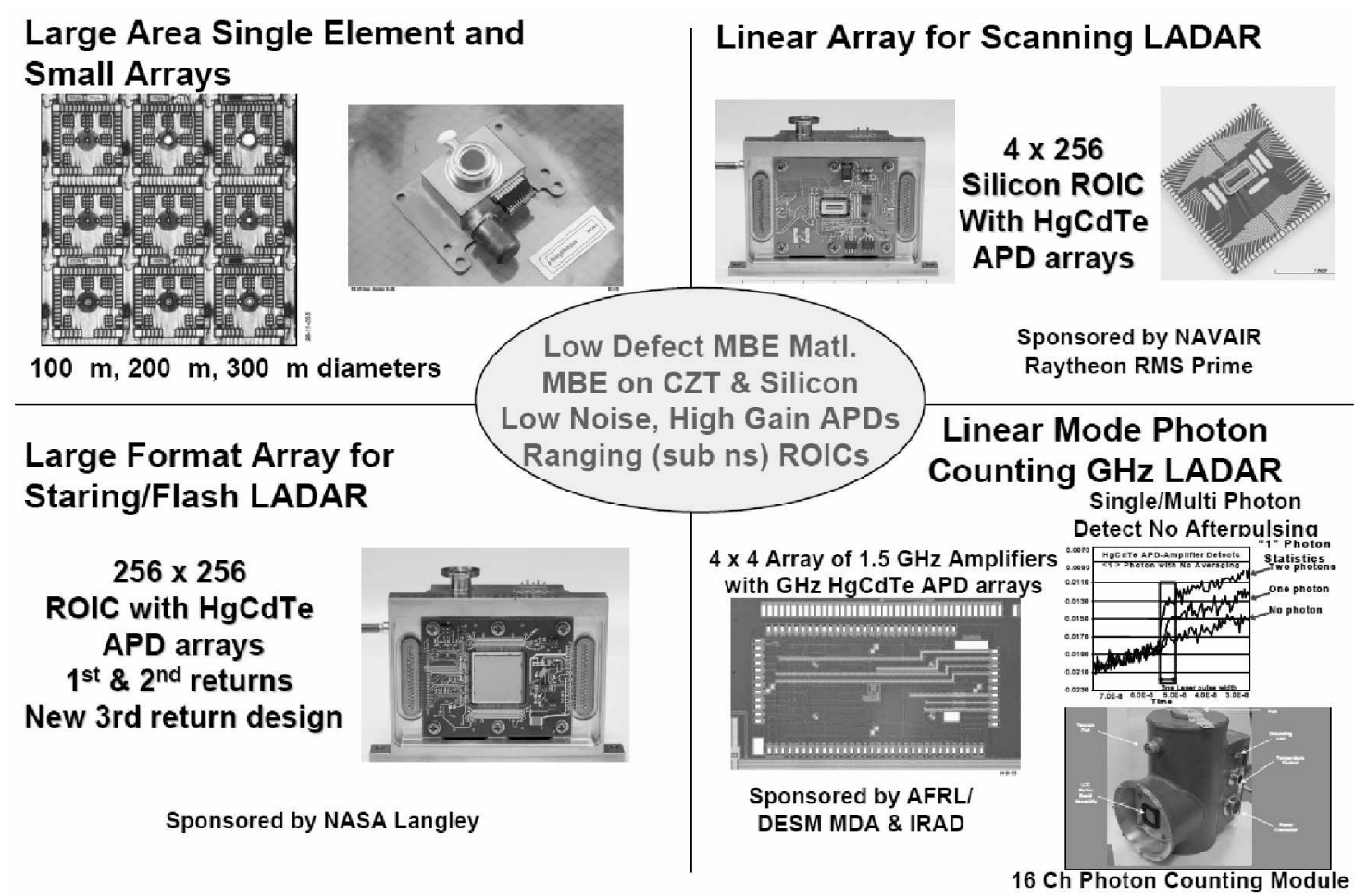

Figure 1. LADAR Products at Raytheon: Include: High Performance Large 100mciron to 300 micron Single Element APDs for Ambient or TEC Operation in the 1-2 micron band: (2) Scanning LADAR sensors $4 \times 256$ configuration; (3) Large Format Flash Sensors $256 \times 256$ Providing up to 3 laser pulse returns with both range and intensity and (4) Photon Counting Modules with Electronics and Dewar

\section{ALHAT PROJECT}

NASA 's Autonomous Precision Landing and Hazard Detection Avoidance Technology (ALHAT) Project is actively pursuing development of 3-D Imaging Flash LADAR technology for precision safe landing on the Moon and other planetary bodies. Future robotic and human exploration vehicles to the Moon will require advanced technology development to support safe and precision landing on the lunar surface. Lunar exploration may require landing in areas of total darkness where hazardous terrain features such as rocks or craters could be obscured. Hazard detection must be quick, achievable under any lighting condition, and at a far enough range to divert and guide the vehicle to a safe landing zone. Although precision-landing accuracies of tens of meters will be required for these missions, the hazard detection sensor will need to resolve terrain features at a few centimeters in-depth from standoff ranges hundreds of meters away. The ALHAT is a NASA project that will develop descent and landing Guidance, Navigation and Control (GNC) hardware and software technologies necessary to advance the state-of-the-art in order to support lunar crew, cargo, or robotic missions.

The primary objective of ALHAT is to develop and mature an autonomous lunar landing GN\&C and sensing system meeting the following general requirements:

- Improve Landing Accuracy from $1 \mathrm{~km}$ to 30 meters

- Incorporate Hazard Detection and Avoidance capability (Detecting rocks $>30 \mathrm{~cm}$ in height, and slopes $>5 \mathrm{deg}$. over $10 \mathrm{~m}$ )

- Enable landing anywhere and under any lighting conditions 
Although ALHAT focuses on lunar landing, its technology can be applied to landing in other planetary bodies in the solar system (e.g. Mars).

Flash LADAR can perform 3 functions critical for precision safe landing:

- Hazard Detection and Avoidance (1000 m to $100 \mathrm{~m})$

- Terrain Relative Navigation ( $15 \mathrm{~km}$ to $2 \mathrm{~km}$ )

- Altimetry (20 km to $100 \mathrm{~m})$

Figure 3 shows the envisioned LADAR configuration. Figure 4 provides the Flash LADAR operational scenario and a description of these functions.

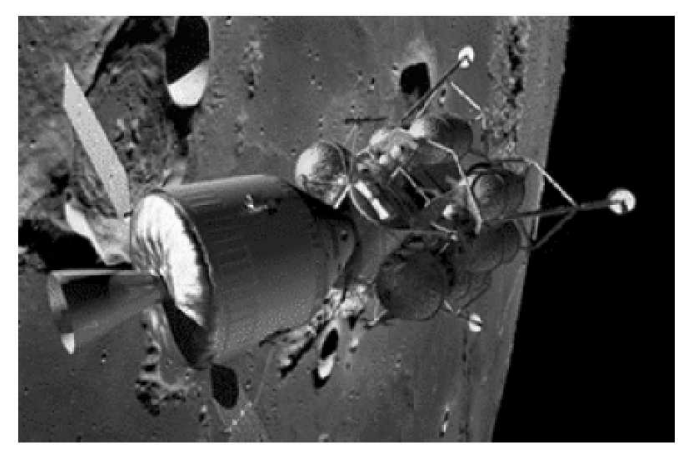

Figure 2 Lander is Shown in Close Proximity to Cratered Lunar or Planetary Surface
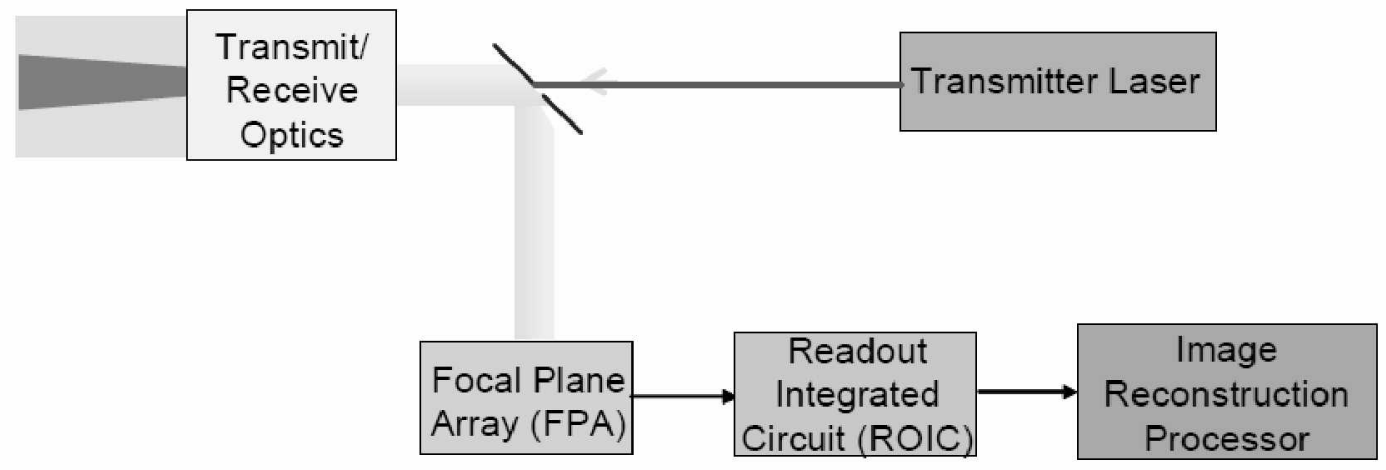

Figure 3. ALHAT LADAR Configuration

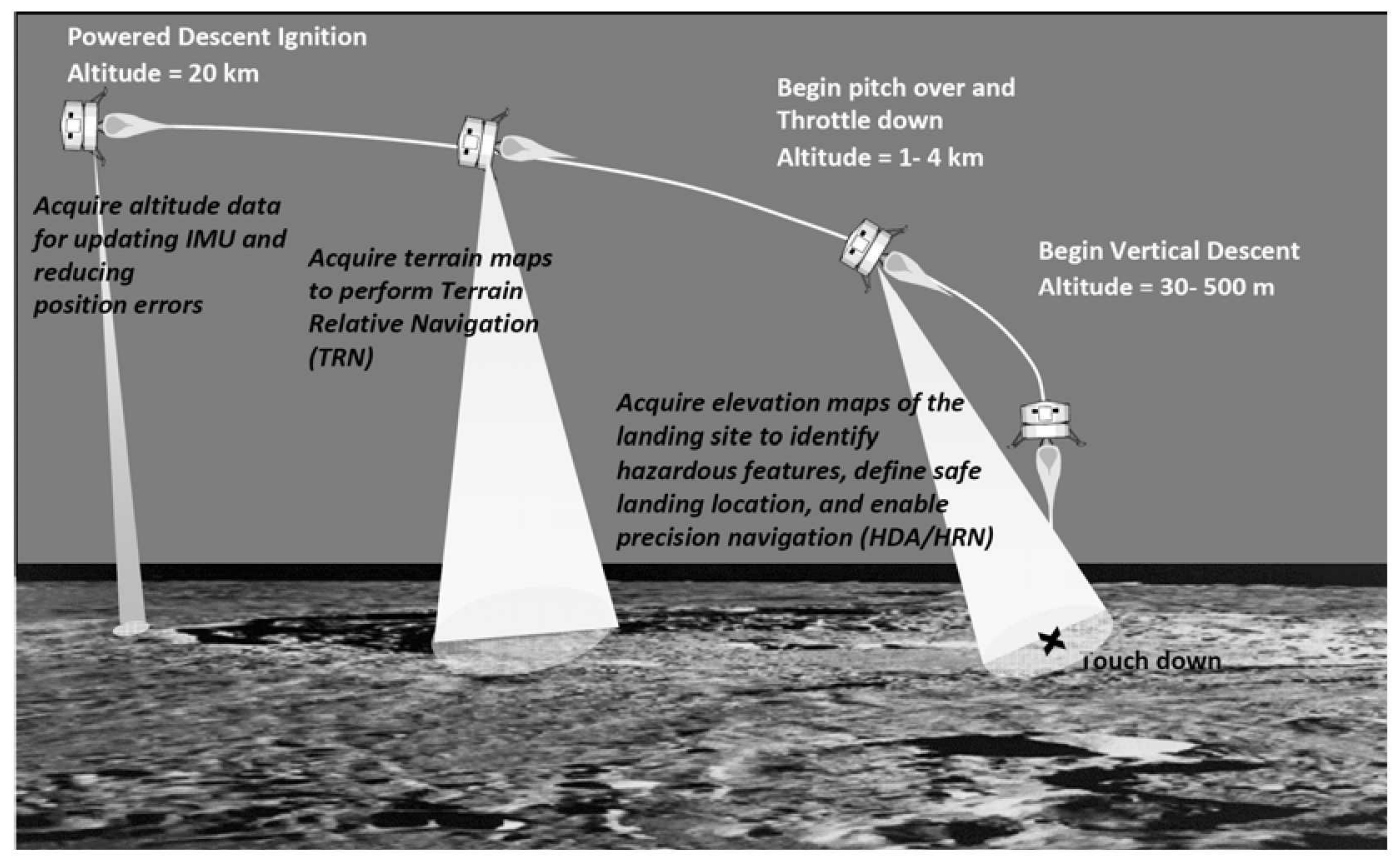

Figure 4. ALHAT Operational Scenario: 3D Flash LADAR is used to acquire altitude data, generate terrain for navigation and acquire precise elevation information identifying hazardous features. This enables precise navigation and landing at a safe location. 
Perhaps the most challenging and important Flash LADAR function is Hazard Detection and Avoidance. Figure 5 illustrates the Flash LADAR operation, during the terminal phase of landing and summarizes its performance goals.

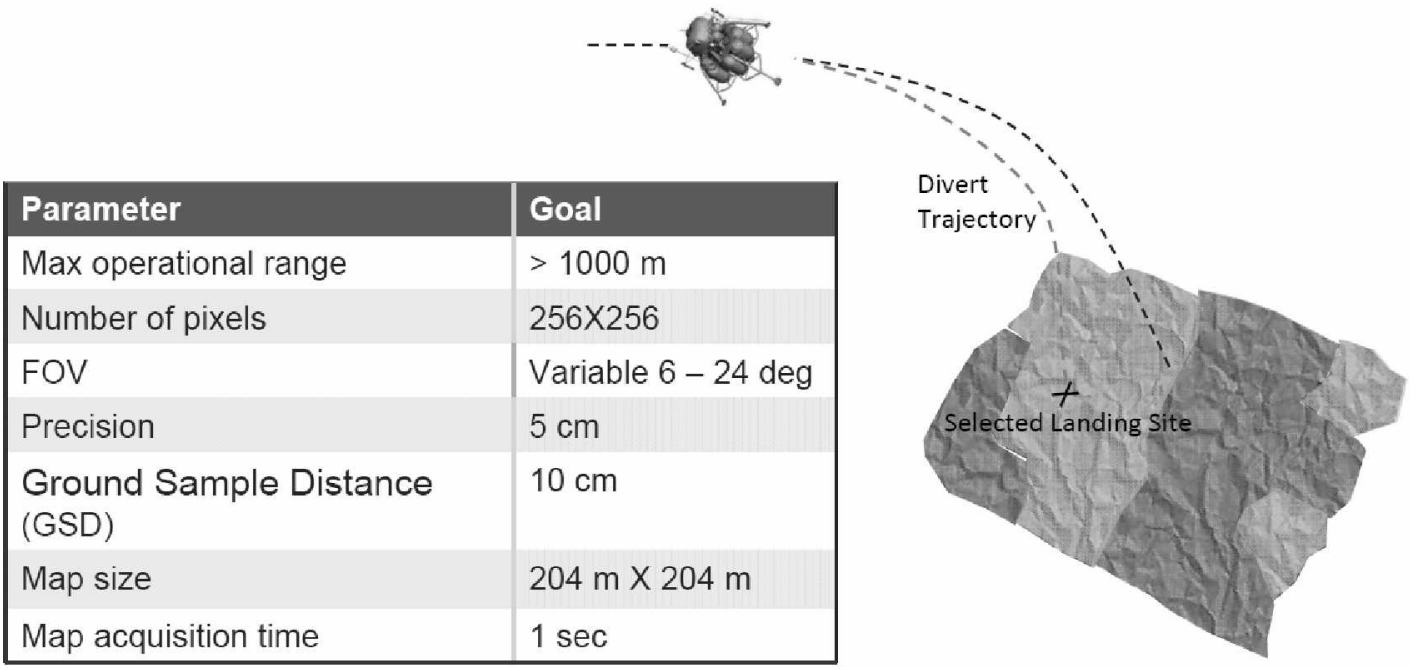

Figure 5. ALHAT LADAR Performance Goals

\subsection{Receiver Operation and Requirements}

3D Data is captured by sensor to support ALHAT mission in the following manner:

1. Narrow Laser Pulse is Fired

2. Sensor is notified on start time

3. Time of return along with intensity of return is captured by circuits

4. Precision of time measurement is $0.3 \mathrm{nS}$ corresponding to a $5 \mathrm{~cm}$ range difference

5. Time of arrival is stored in each pixel

6. Intensity of return is stored in each pixel

7. Both time of arrival and intensity data stored in each pixel is readout at $60 \mathrm{~Hz}$ Rate

8. Providing a updated 3D Image every $1 / 60$ th of a second to the HDEM

To achieve ALHAT LADAR performance goals we have established key detector \& ROIC requirements summarized in Table 1 and Table 2, respectively:

Table 1. Key Detector Requirements

\begin{tabular}{|c|c|c|c|c|c|c|c|}
\hline $\begin{array}{c}\text { Array } \\
\text { Format }\end{array}$ & $\begin{array}{c}\text { Pixel Size } \\
(\mu \mathrm{m})\end{array}$ & $\begin{array}{c}\text { Pixel Pitch } \\
(\mu \mathrm{m})\end{array}$ & $\begin{array}{c}\text { Quantum } \\
\text { Efficiency }(\%)\end{array}$ & $\begin{array}{c}\text { Wavelength } \\
\text { Optimization }(\mu \mathrm{m})\end{array}$ & Gain & $\begin{array}{c}\text { Bandwidth } \\
(\mathrm{GHz})\end{array}$ & $\begin{array}{c}\text { Excess } \\
\text { Noise }\end{array}$ \\
\hline $256 \times 256$ & 60 & 60 & 70 & 1.5 & 20 & 1 & 2 \\
\hline
\end{tabular}

Table 2. ROIC Requirements

\begin{tabular}{|c|c|c|c|c|c|c|}
\hline Format & $\begin{array}{c}\text { Cell Size } \\
(\mu \mathrm{m})\end{array}$ & $\begin{array}{c}\text { Cell Pitch } \\
(\boldsymbol{\mu m})\end{array}$ & $\begin{array}{c}\text { ROIC Noise } \\
(\mathbf{p A} / \mathbf{r t}-\mathrm{Hz})\end{array}$ & $\begin{array}{c}\text { Dynamic Range } \\
(\mathrm{bits})\end{array}$ & $\begin{array}{c}\text { Range Resolution at } \\
\mathbf{1 ~ k m}(\mathbf{c m})\end{array}$ & $\begin{array}{c}\text { Frame Rate } \\
(\mathbf{H z})\end{array}$ \\
\hline $256 \times 256$ & 60 & 60 & 0.5 & 12 & $5(0.33 \mathrm{~ns})$ & 30 \\
\hline
\end{tabular}




\subsection{ALHAT Receiver Component}

Figure 6 identifies the key components of Raytheon's ALHAT LADAR Building blocks of the $256 \times 256$ Array Receiver Assembly. These include: HgCdTe APDs grown by MBE and processed into $256 \times 256$ arrays. APD design has been optimized for Fex $\sim 1$ and high gain at ambient temperature. Readout Integrated Circuits fabricated on 6" Silicon wafers designed through extensive modeling and simulation to achieve performance listed in Table 2 and a compact low profile package. Not shown is compact electronics for operation of the hybrid chip

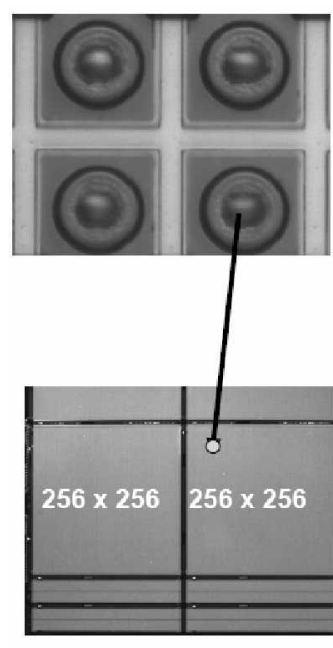

APD Arrays

\section{Low noise $\mathrm{HgCdTe}$} APD diodes

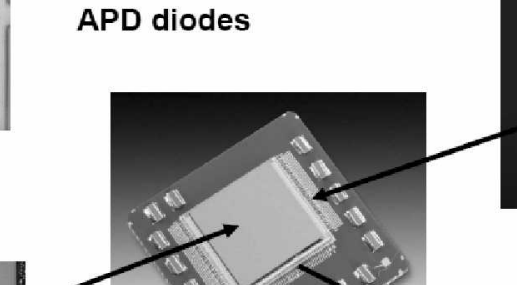

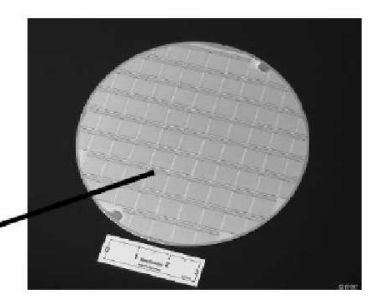

Readout Integrated Circuits Wafers

Figure 6. Key Components of the ALHAT $256 \times 256$ LADAR Receiver

\section{HGCDTE AVALANCHE PHOTODIODES}

\subsection{MBE Growth}

Figure 7 illustrates $\mathrm{HgCdTe}$ arrays grown by MBE using an NIP configuration and processed into arrays.

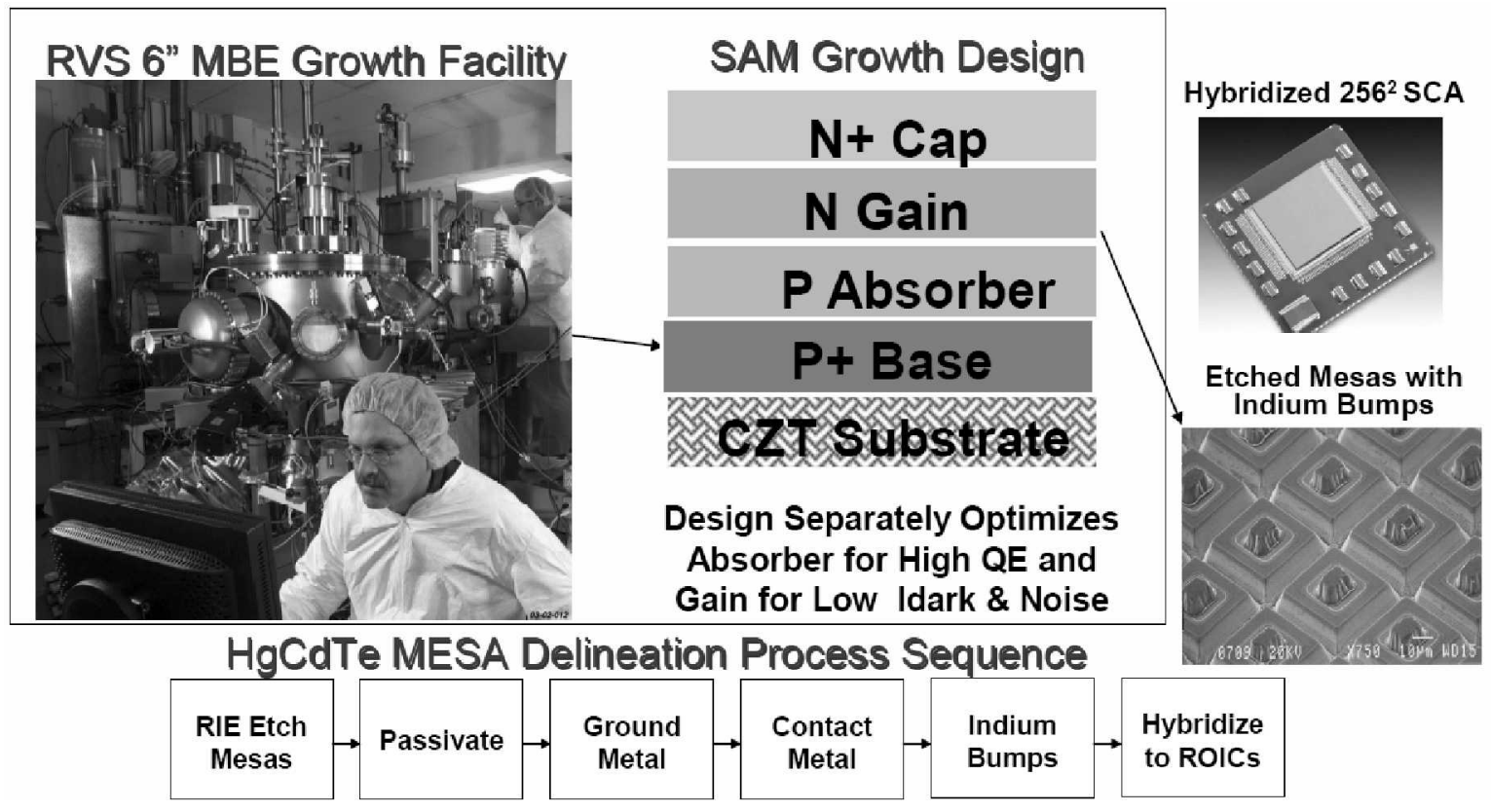

Figure 7. HgCdTe APD Design and Molecular Beam Epitaxial Growth \& Processing 
Key to successful growth of MBE layers is the precise deposition on an atomic scale of the matrix elements (i.e. Mercury, Cadmium, and Tellurium) as well as the dopant atoms Arsenic and Indium. This control is enabled by several tools including Spectral Ellipsometry which continuously monitors the surface and measures the HgCdTe composition and provides real-time feedback to the rate control of the sources the $\mathrm{HgCdTe}$ composition. With careful control of temperature and rates throughout the deposition cycle defect densities of $<500$ microdefects per cubic centimeter have been routinely achieved for APD layer growth on Cadmium Zinc Telluride $\left(\mathrm{Cd}_{\mathbf{x}} \mathrm{Zn}_{1-\mathbf{x}} \mathrm{Te}\right)$ substrates. This defect density is state of the art.

\subsection{Building APD Arrays}

Once the layer is grown they are processed utilizing the steps outlined in Figure 7. The delineated $256 \times 256$ arrays are shown by photo-microscopy in Figure 8. The typical CZT wafer can hold four full arrays. Figure 9 shows a wafer that includes test structures and smaller $256 \times 16$ arrays and test structures. Wafer probe measurements are made at selected sites shown at the edges of the arrays to assess lot and wafer performance. These measurements provide feedback on the design, growth and process steps. Typical characteristics measured include parameters such as total dark current, gain normalized dark current and excess noise versus gain or voltage. Also gain versus voltage. Given the same growth and processing steps values we measure are closely matched from lot to lot and wafer to wafer.

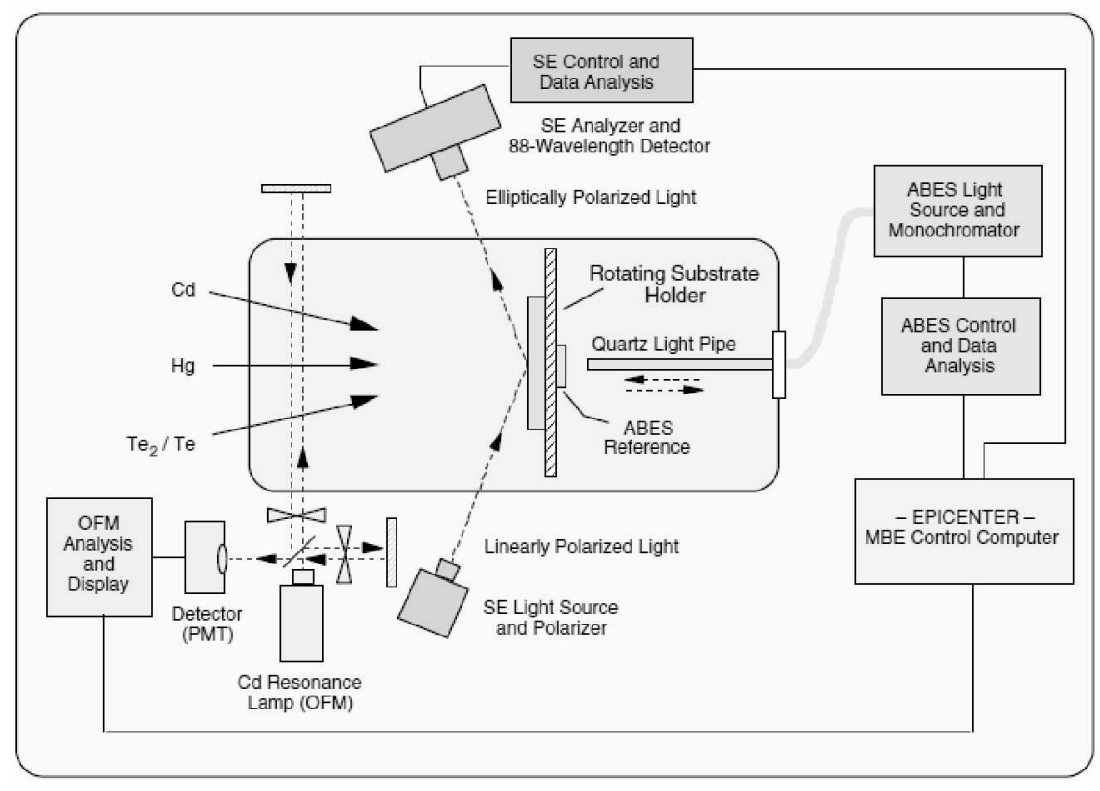

Figure 8. HgCdTe MBE Systems Fitted with In-Situ Sensors for Precise Alloy Composition \& Substrate Temperature Control. 


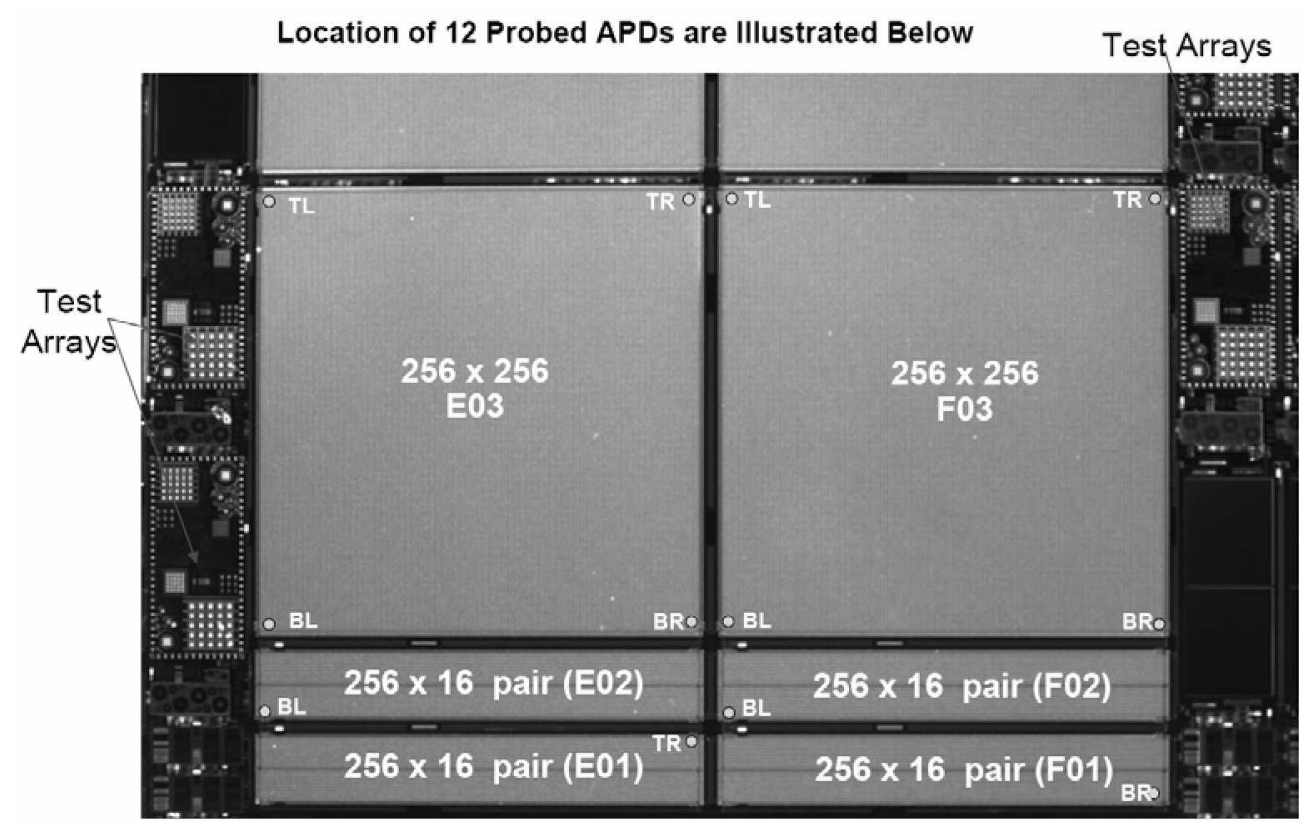

Figure 9. Full Processed Wafer Showing 2 Large $256 \times 256$ APD Arrays and 4 Pairs of $256 \times 16$ APD Arrays

\subsection{Manufacturing In-Process Tests}

Tests of all key components during the manufacturing process enable high confidence selection of components meeting performance specification and reduce cost to the customer. For example wafer evaluation after MBE growth eliminates high defect wafers. Wafers are screened after processing to select good arrays and provide feedback to growers and processing personnel. Statistical wafer measurements provided a preliminary data on uniformity of key APD parameters such as gain at a fixed bias. Similarly ROIC wafers are screened at wafer probe to identify fully functional ROIC die. Hybridized Sensor Chip Assemblies (SCAs) are evaluated for full functionality and operability prior to installation in the integrated dewar assembly.

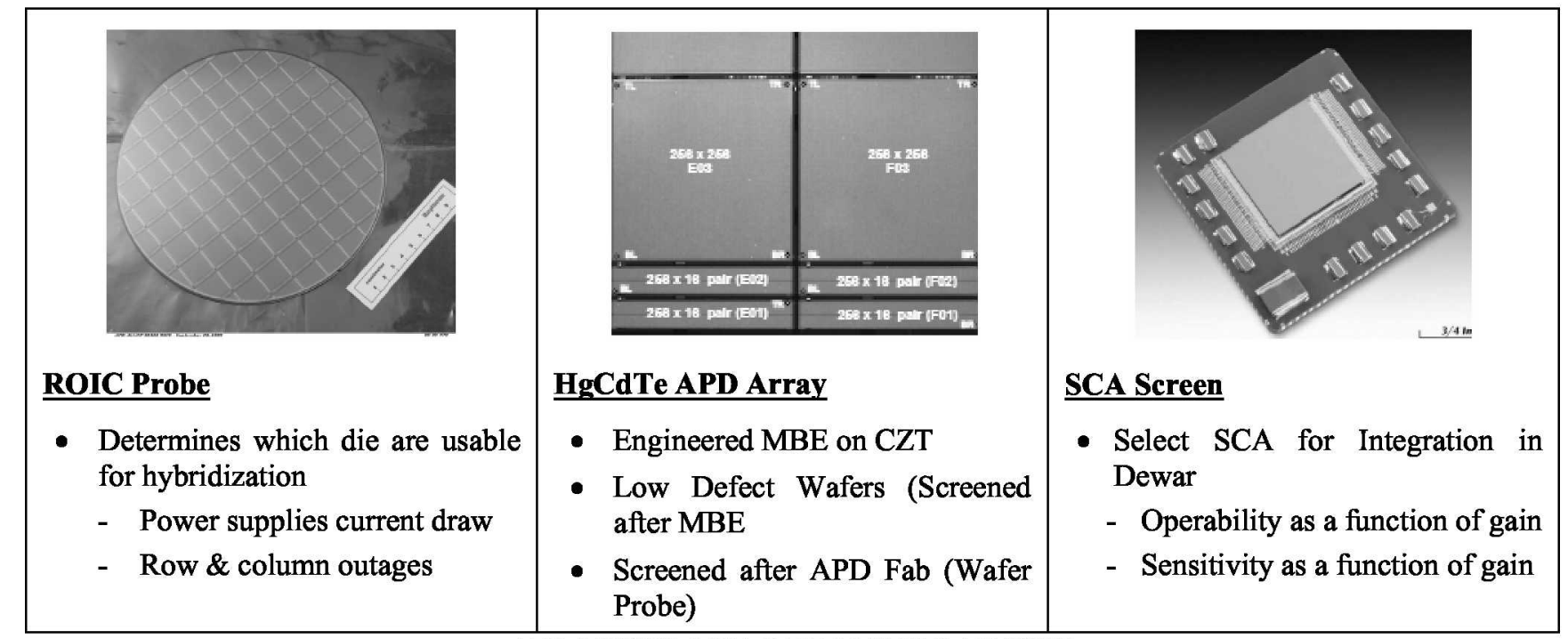

\section{ALL TESTS AND OPERATION AT 300K}

Figure 10. Manufacturing In-Process Tests 


\subsection{APD Wafer Measurements}

Figure 11 shows typical ambient temperature wafer probe measurements on processed APDs. Key parameters measured include: Gain vs. Voltage, Idark and Idark normalized to gain vs. gain, Excess noise and Fex and NEP with $42 \mathrm{MHz}$ bandwidth. Below is a typical set of parameter measurements vs. gain or voltage from an ALHAT wafer. APDs were tested to a gain of 100 and show excellent Idark normalized to gain of $<10 \mathrm{nA}$, excess noise Fex $\sim 1$ at all gains and NEP $\sim 0.5 \mathrm{nW}$.
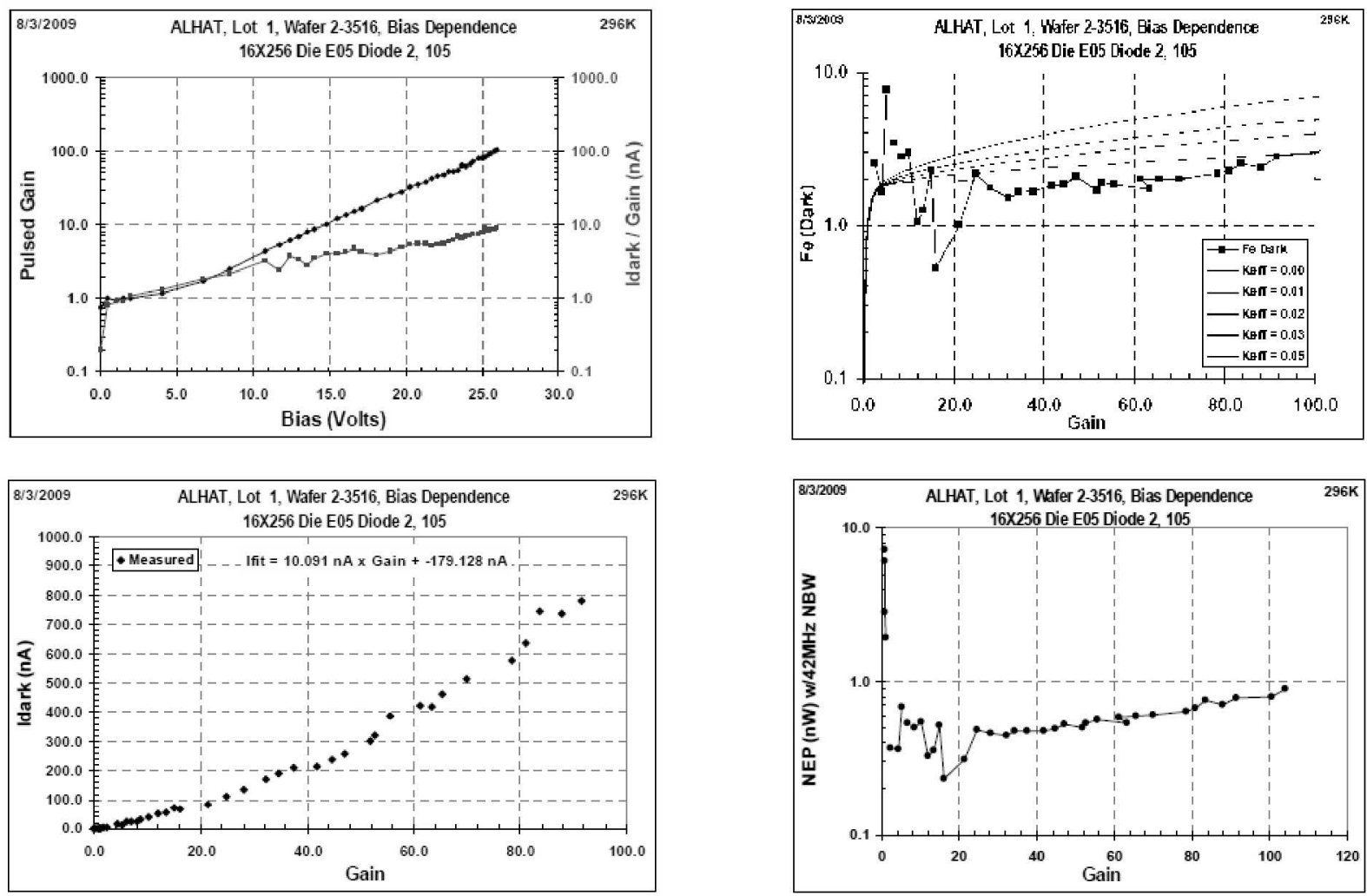

Figure 11 APD Wafer Measurements at 300K

\subsection{Statistical Wafer Measurements}

Figure 12 illustrates the protocol selected for statistical measurements on the wafer. Forty five diodes were tested every fifth diode along a row that spans 256 APDs. Gain, responsivity, dark current and NEP were extracted. Figure 13 shows a skyline plot of all 45 diodes at various bias voltages selected to achieve gains of 5, 10, 20, 40, 60,80 and 100 . All APDs tested had full operability and achieved a gain of 100 . As seen on small variations in gain along the row along the row of 45 diodes is observed for each gain from 5 to 100 . Non-uniformity (sigma/mean) of $<8 \%$ was seen for all gains. With low defect wafers now being grown this excellent performance is routinely seen on multiple wafers. 
- Bias dependence data taken on 45 diodes

- Detector bias voltage stepped

- Signal and noise measurements

- Test used to derive excess noise factor

- Data extracted and plotted

- Gain

- Responsivity

- Dark Current

- NEP@ $42 \mathrm{MHz}$ bandwidth

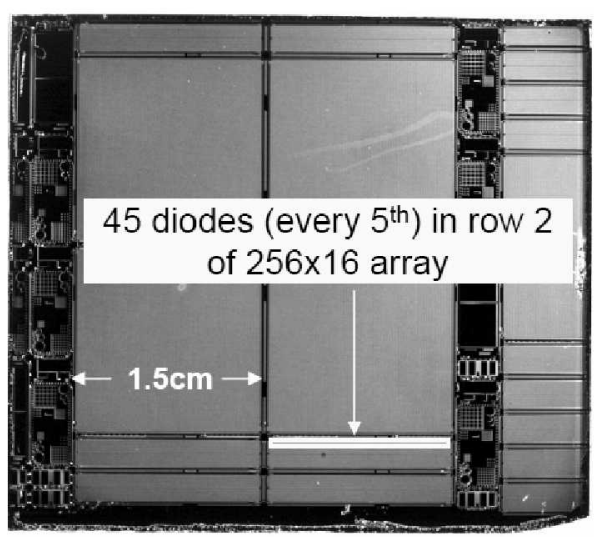

Figure 12. Statistical APD Data Derived From Probe Test of Wafer

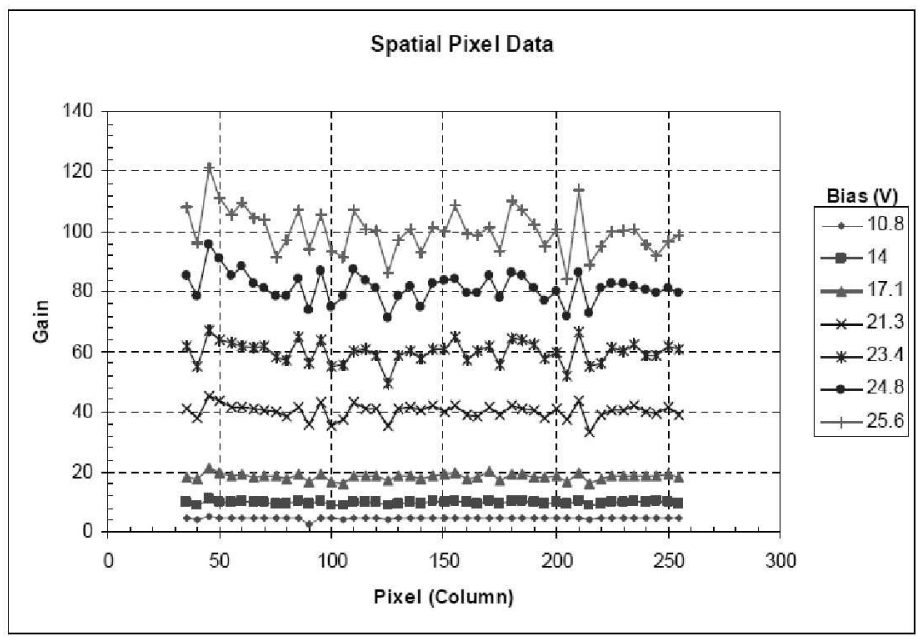

Figure 13. Wafer Probe Data Showing Good Uniformity and $100 \%$ Operability at Fixed Bias at to Gain of 100 Comparable high yields are seen for multiple wafers

\section{READOUT INTEGRATED CIRCUITS, SCAS AND TEST DATA}

\subsection{ROICs}

The ALHAT $256 \times 256$ Readout integrated circuit (ROIC) is a 4th generation design. It has been optimized for performance required by NASA for the lunar landing. The design utilizes a 0.18 micron CMOS process to provide sophisticated functionality per pixel while achieving the desired very low noise $0.5 \mathrm{pA} / \mathrm{rt}-\mathrm{Hz}$ to complement the APD performance. A portion of an 8" wafer is seen in Figure 14. Laser returns are detected asynchronously (not gated) and both intensity and time of arrival are acquired for up to three returns per frame time. Timing precision is key to accurate relative range data i.e. range precision. To achieve required $5 \mathrm{~cm}$ range precision the ROIC timing accuracy has been improved to 300ps. Multiple returns from a single outgoing pulse are expected when a pixel sees the edge of a boulder and the adjacent ground. Each pixel can capture up to three returns determining and storing intensity and time of arrival for each. 
- $256 \times 256$ format

- 0.18 micron CMOS

- 60 micron pixels

- Asynchronous acquisition of laser returns (not gated)

- Low noise amplification, $0.5 \mathrm{pA} / \mathrm{rt}-\mathrm{Hz}$

- Range and intensity acquired for up to 3 returns

- Range precision $5 \mathrm{~cm}(0.3 \mathrm{~ns})$

- $60 \mathrm{~Hz}$ operation

Figure 14 ALHAT ROICs

\subsection{Packaged SCAs}

Once functioning ROIC die and detector die have been identified, they are "Indium bump hybridized" using the process presently utilized for IR sensors. Figure 15 shows the hybridized Sensor Chip Assembly (SCA) attached and bonded into a Leadless Chip Carrier preparatory to functional testing. Passive components are installed on the same package to optimized performance.
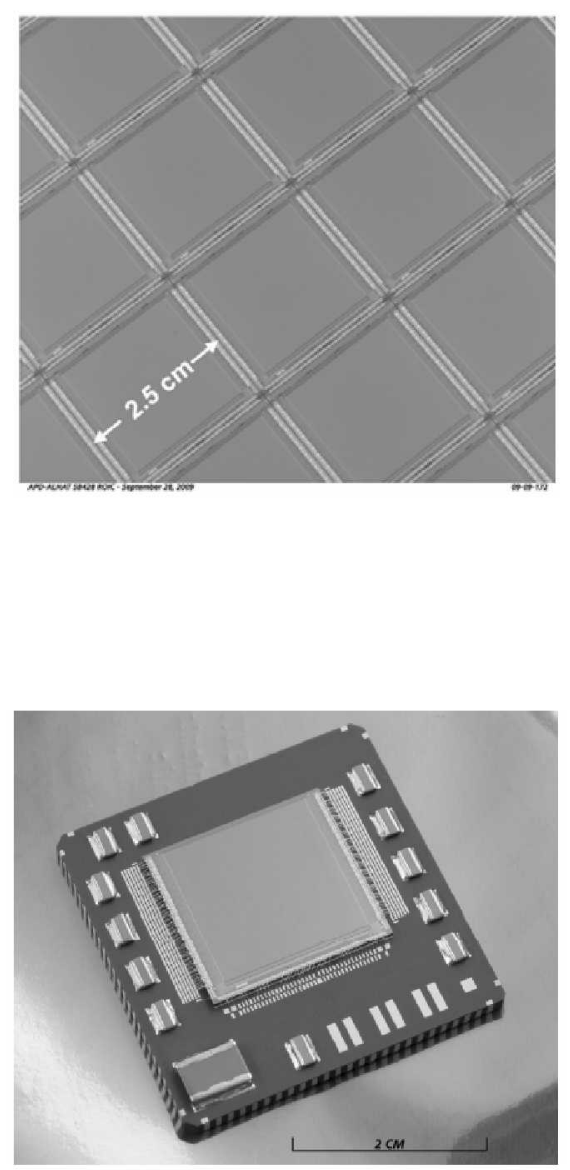

Figure 15. ALHAT SCA

\subsection{SCA Test Results}

SCAs are evaluated for full functionality and quantitative measurements are performed across the entire array of key parameters which include signal response, responsivity, noise and NEP. A key sensor/system parameter, Minimum Detectable Signal is also determined. The following is typical data from an SCA candidate for delivery.

Figure 16 shows results from a uniform laser illumination of the SCA. The operability at unity gain and 300K is shown in this figure at a reverse bias of 1volt. Note that full response on our HgCdTe APDs is achieved at low voltage. Excellent operability $\mathbf{9 9 . 7 \%}$ is observed with scattered bad pixels. Good flat-field uniformity can be seen.

Figure 17 quantifies the responsivity at a gain of 20 at a nominal gain of 20 . Responsivity with a 1.55 micron laser illumination is $>15 \mathrm{~A} / W$. This is for an SCA without antireflection coating. Good uniformity with operability $>97 \%$ is seen. 


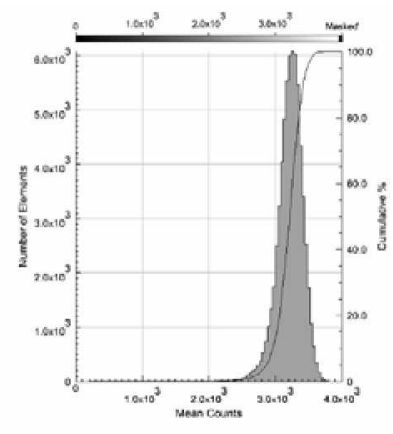

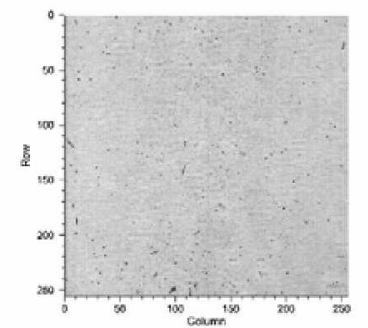
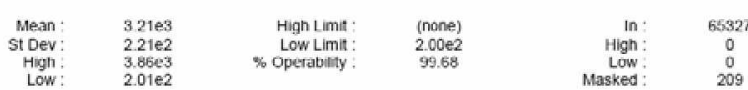

Figure 16. Signal Operability At Unity Gain at $\mathbf{T}=$ $300 \mathrm{~K}$ and Vbias $\sim 1$ volt is $99.7 \%$
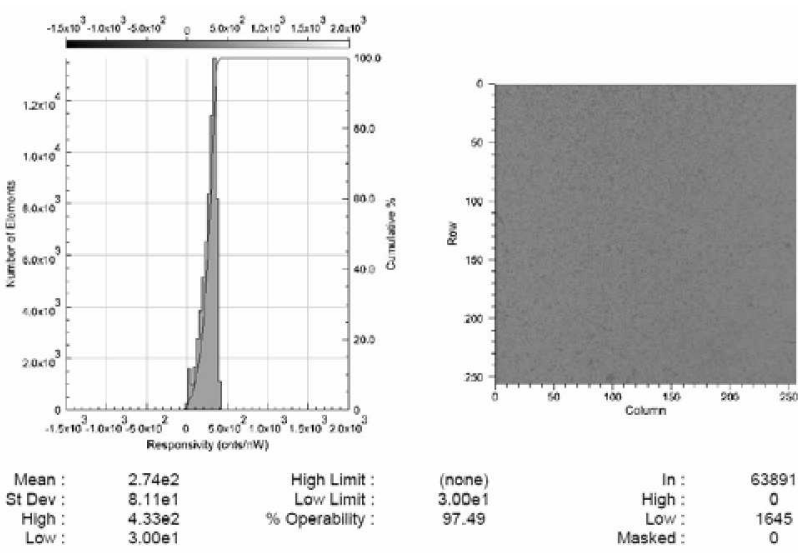

Figure 17. Responsivity SCA Map at Average Gain of Twenty

Noise is measured for the array and the NEP calculated as the quotient of noise divided by responsivity. Array image shown in Figure 18 shows again a very tight distribution with scattered noisy pixels. Good uniformity with operability $\sim$ $97 \%$ is seen
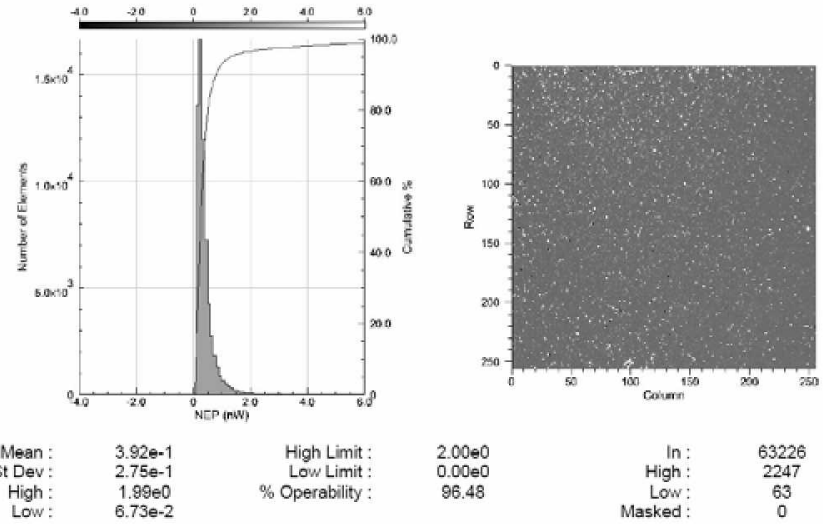

Figure 18 NEP SCA Array Map at Gain $=20$ at $300 \mathrm{~K}$

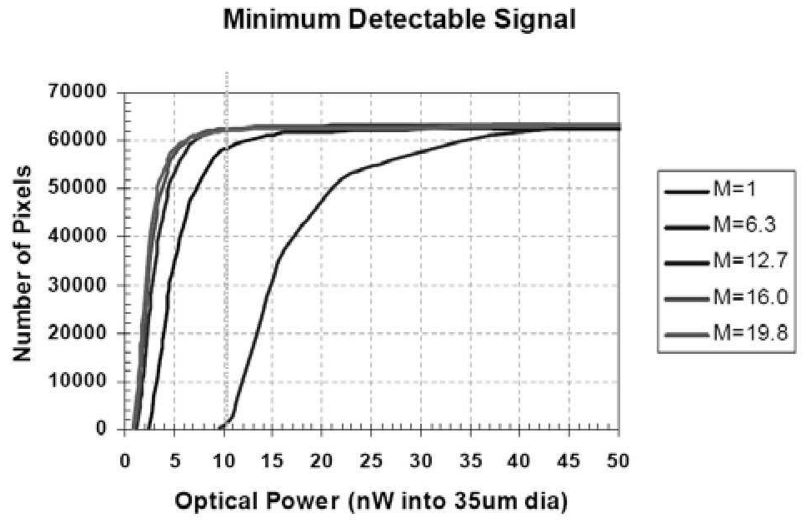

Figure 19. Minimum Detectable Signal vs. Gain (Room Temp) MDS Meets Goal of $<10 \mathrm{nW}$

Minimum Detectable Signal is a key system-sensor parameter and corresponds to array level signal where high probability of target detection can be achieved. This is shown in Figure 19 as a function of detector bias, hence average gain. The MDS definition is the signal level at which $95 \%$ of the array triggers on the return laser pulse 95 times or more out of 100 laser shots. The plots cumulative number of pixels which detect the laser pulse $95 / 100$ times as the laser pulse energy is raised. If we determine MDS when it meets the horizontal line we see that the MDS for the array decreases from unity gain value of $45 \mathrm{nw}$ to a $15 \mathrm{nW}$ at a gain of 6 to $\sim 5 \mathrm{nW}$ at a gain of $15-20$, clearly meeting the design goal of $<10 \mathrm{nW}$. Note also that the MDS continues to improve as the gain is increased in contrast with III-V APDs where increases in gain at some point will increase the noise due to excess noise factors. The good performance is due to the near unity excess noise factor for $\mathrm{HgCdTe}$.

\section{COMPACT PACKAGE AND ELECTRONICS}

The ability of Raytheon's HgCdTe APD array based LADAR receivers to operate at room temperature allows a very low cost, compact approach to packaging. Figure 20 shows the hermetic package including electronics. The package utilizes 
only a single stage inexpensive Thermal-Electric Cooler (TEC) to stabilize the APD temperature to approximately $300 \mathrm{~K}$. Package size is approximately 3" diameter by about 3" in depth. It provides the temperature stabilization, regulated power and electronic clocks and biases needed to run the chip, SCA programming needed to establish pixel level thresholds, and the first level amplification of the multiplexed outputs which provide the time of arrival (range) and intensity for up to 3 returns per each of the 65000 pixels.

\section{Inputs}

- Controlled TEC Power

- Regulated SCA Power

- SCA Clocks
- SCA Programming

- Pixel Threshold Settings

- Laser Light

\section{Outputs}

- Multiplexed Analog Pixel - SCA Temperature Data

- Intensity

- Time of Arrival

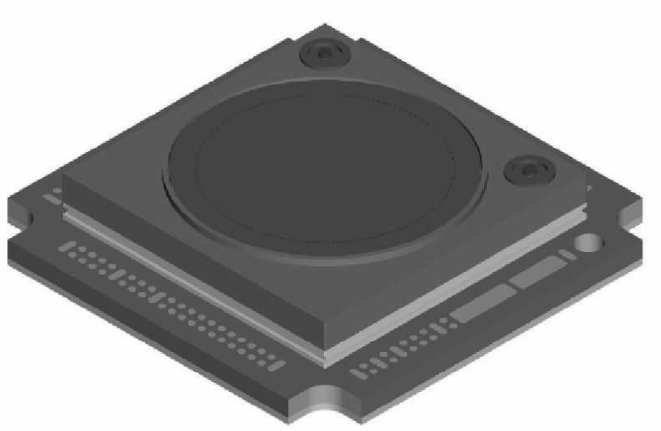

Figure 20. ALHAT Detector Assembly

\section{SUMMARY AND PLANS}

Table 3 summarizes key points of the paper:

Table 3. Key Accomplishments

\section{Large Low Defect MBE APD Wafers Grown}

- Low Defects $<500 / \mathrm{cm}^{2}$ on $5 \times 5 \mathrm{~cm} \mathrm{CdZnTe} \mathrm{Wafer}$

- Detector Array Size $2 \times 2 \mathrm{~cm}$

\section{APDs Achieve Gains Up to 100 at Wafer Probe 300K}

- $100 \%$ Operability

- Idark/Gain $<10 \mathrm{nA}$

- $\quad$ Fex $\sim 1$

- Very Uniform at High Gains ( $\sigma /$ mean $\sim 8 \%$ across Wafer

\section{$256 \times 256$ ROIC Provides Range and Intensity for Up to Three Returns}

- Asynchronous Acquisition Laser Pulses (Not Gated)

- $5 \mathrm{~cm}$ Range Precision

- $\quad<0.5 \mathrm{nA} / \mathrm{rt}-\mathrm{Hz}$ Noise Floor

$256 \times 256$ SCA Has Excellent Operability at Ambient Temperature

- Responsivity $>15 \mathrm{~A} / \mathrm{W}$

- $\quad \mathrm{NEP} \sim 0.4 \mathrm{nW}$

- Minimum Detection Signal for 655.36 Detectors is $<10 \mathrm{nW}$

Raytheon has developed a suite of high performance HgCdTe APD and LADAR Receivers including high performance single large APDS, scanning LADAR sensors and systems, large format Flash LADAR (the focus of this development) and sub photon sensitivity Linear Mode Photon Counting SCAs and modules 
A common element is that they utilize low defect MBE grown HgCdTe APDs which provide gains up to 100 at Ambient Temperatures with good dark current Idark/gain $<10 \mathrm{nA}$ and Fex $\sim 1$.

Sensors utilize a sophisticated 4th generation ROIC design which provides asynchronous multipulse detection (up to 3) with capture of time of arrival and intensity for each. Very low noise $-0.5 \mathrm{pA} / \mathrm{rt}-\mathrm{Hz}$ and excellent range precision $5 \mathrm{~cm}$ is achieved using the 0.18 micron CMOS process.

Careful measurements show that the detectors, ROIC and SCAs built for the ALHAT program meet NASA's mission requirements with SCA performance exhibiting excellent uniformity, operability $>97 \%$ at high gains $\sim 20$, average NEP $<0.4 \mathrm{nW}$ and $\mathrm{MDS}<10 \mathrm{nW}$ all at ambient temperature.

RVS capability in advanced LADAR receives has been successfully applied to meeting the requirements for NASA's ALHAT Mission specifically a Robust multifunctional 2562 3D Imaging Sensor for navigation, lunar map generation and safe lunar landing site determination. The integrated receiver with electronics will be delivered to NASA in Q4 of 2010. It is anticipated that the LADAR receiver technology demonstrated will have multiple applications in supporting NASA and DOD missions.

\section{ACKNOWLEDGEMENTS}

The authors Steven Bailey, William McKeag, Jinxue Wang, and Michael Jack from Raytheon Vision Systems and Farzin Amzajeridan from NASA Langley wish to acknowledge critical technical and administrative support from the following Raytheon LADAR and NASA ALHAT team members:

\section{Raytheon Vision Systems (Santa Barbara, CA)}

- J. Ashbrock

- G. Chapman

- G. Crawford

- E. Herrin

- C. Hoyt

- I. Kasai
- V.Lee

- K. Liu

- M. Mitani

- J. Wehner
Raytheon Missile Systems (Tucson, AZ)

- T. Roberts

- J Neisz

HRL Labs LLC (Malibu, CA)

- T. DeLyon 